\title{
Prevenção de lesão de septo nasal em neonatos pré-termo: revisão integrativa da literatura
}

\author{
Preventing lesions of nasal septum in preterm newborns: an integrative literature review
}

Prevención de lesión de septo nasal en neonatos prematuros: revisión integrativa de la literatura

Suely de Fátima Santos Freire Bonfim ${ }^{1}$, Simone Maria Muniz da Silva Bezerra², Maria Gorete Lucena de Vasconcelos ${ }^{3}$, Luciana Pedrosa Leal ${ }^{4}$

1 Enfermeira, Mestre em Enfermagem. Enfermeira da Unidade de Terapia Intensiva Neonatal do Hospital das Clínicas da Universidade Federal de Pernambuco (UFPE). Recife, PE, Brasil. E-mail: sfbonfim@uol.com.br.

${ }^{2}$ Enfermeira, Doutora em Fisiologia Geral. Professora Adjunta da UFPE. Recife, PE, Brasil. E-mail: simonemuniz@yahoo.com.br.

${ }^{3}$ Enfermeira, Doutora em Enfermagem em Saúde Pública. Professora Associada da UFPE. Bolsista de Produtividade em Pesquisa do CNPq - Nível 2. Recife, PE, Brasil. E-mail: mariagoretevasconcelos@gmail.com.

${ }^{4}$ Enfermeira, Doutora em Nutrição. Professora Adjunta da UFPE. Recife, PE, Brasil. E-mail: lucianapleal@hotmail.com.

\section{RESUMO}

Este estudo objetivou investigar as evidências científicas relacionadas aos cuidados de enfermagem na prevenção de lesão de septo nasal em pré-termos em ventilação utilizando prongas. Foi realizada uma revisão integrativa da literatura nas bases eletrônicas LILACS, BDENF, CINAHAL, PUBMED e na biblioteca SciELO. Foram selecionados nove estudos entre 2000 e 2013, disponibilizados on-line ou pelo autor, publicados em português, inglês e espanhol. Os resultados evidenciaram que $70 \%$ dos estudos abordam cuidados ligados à qualidade e adequação dos dispositivos nasais e à vigilância contínua da equipe de enfermagem. A inspeção do septo e columela nasal, proteção com hidrocoloide e a instalação adequada do sistema foram considerados cuidados importantes para a prevenção de lesões. Conclui-se que existe necessidade de adequação dos cuidados de enfermagem e monitorização contínua do dispositivo e do neonato, durante a ventilação, para a prevenção das lesões de septo nasal.

Descritores: Pressão Positiva Contínua nas Vias Aéreas; Prematuro; Ferimentos e Lesões; Cuidados de Enfermagem.

\section{ABSTRACT}

The goal of this study is to investigate scientific evidence related to nursing care for preventing lesions of the nasal septum in preterm infants who are using nasal prong ventilation. It is an integrative literature review through LILACS, BDENF, CINAHAL, PUBMED and SciELO library electronic databases. We selected 09 studies between 2000 and 2013 , available online or through the author, published in Portuguese, English and Spanish. The results displayed that $70 \%$ of the studies examine the quality and adaptation of nasal devices and the nursing team's continual vigilance. Septum and nasal columella inspection, hydrocolloid protection and adequate system installation were considered important factors which contribute towards preventing lesions. We conclude that the adaptation of nursing care and constant monitoring of the device and of the newborn during ventilation are necessary to prevent nasal septum lesions.

Descriptors: Continuous Positive Airway Pressure; Infant, Premature; Wounds and Injuries; Nursing Care.

\section{RESUMEN}

Se objetivó investigar las evidencias científicas relativas a cuidados de enfermería en prevención de lesiones de septo nasal en prematuros ventilados mediante cánulas. Se realizó una revisión integrativa de literatura en las bases informáticas LILACS, BDENF, CINAHL, PUBMED y en la biblioteca SciELO. Fueron seleccionados 9 estudios, entre 2000 y 2013, disponibilizados online o por el autor, publicados en portugués, inglés y español. Los resultados evidenciaron que $70 \%$ de los estudios abordan cuidados vinculados a la calidad y adecuación del dispositivo nasal y la vigilancia continua del equipo de Enfermería. La inspección del septo y columela nasal, protección con hidrocoloide e instalación adecuada del sistema fueron consideradas cuidados importantes para prevención de lesiones. Se concluye en que existe necesidad de adecuación de cuidados de enfermería y monitoreo continuo del dispositivo y del neonato durante la ventilación, para la prevención de lesiones de septo nasal.

Descriptores: Presión de las Vías Aéreas Positiva Contínua; Prematuro; Heridas y Traumatismos; Atención de Enfermería. 


\section{INTRODUÇÃO}

A elevada taxa de nascimentos prematuros tem aumentado a utilização do método de ventilação não invasiva como alternativa terapêutica para essa clientela ${ }^{(1)}$. Esse método é destinado ao fornecimento de pressão positiva contínua em vias aéreas, por meio de um duplo tubo curto de material em silicone denominado de pronga nasal, conectado diretamente às narinas do neonato e as conexões do sistema ligadas a um ventilador mecânico ou a um sistema de selo d'água(2).

A pressão exercida pelo dispositivo, na maioria das vezes, leva a ocorrência de lesão de septo nasal no neonato. Lesões pelo uso inadequado de prongas podem variar de uma simples hiperemia de mucosa nasal, sangramento, formação de crostas até a destruição total da columela ${ }^{(3-4)}$ e do septo nasal ${ }^{(2,5)}$.

A imaturidade do sistema tegumentar dos recémnascidos (RNs) os torna mais susceptíveis ao aparecimento de erosões cutâneas e bolhas como resposta aos mínimos traumas(6). Tem sido descrito na literatura incidências de trauma nasal secundário ao uso de pressão positiva contínua em vias aéreas em torno de $20 \%$ a $60 \%$, representando um potencial evento adverso, com consequências em curto prazo como hiperemia nasal e desenvolvimento de lesões e a longo prazo com perda total do septo nasal ${ }^{(1,7-9)}$.

Os cuidados prestados aos RNs submetidos à ventilação não invasiva na prevenção das lesões de septo nasal são realizados em grande parte pela equipe de enfermagem. Ademais, a enfermagem responde por vários mecanismos de prevenção, seja por meio de atividades administrativas, de supervisão, treinamento de pessoal ou nos cuidados prestados aos neonatos admitidos na Unidade de Terapia Intensiva (UTI) neonatal(10). Tal problemática vem despertando nos profissionais que assistem a essa clientela e, em especial na equipe de enfermagem, inquietação e interesse no aprofundamento do tema.

Apesar da assistência especializada aos RNs em UTI, a alta incidência de lesões nasais indica a fragilidade, seja por inadequação ou lacunas nos cuidados dispensados. Dessa forma, reunir evidências atuais sobre os cuidados necessários na prevenção de lesão de septo nasal em RNs pré-termos trará subsídios para a prática da Enfermagem.

Dentro dessa perspectiva, o estudo teve como objetivo investigar as evidências científicas relacionadas aos cuidados de enfermagem na prevenção de lesão de septo nasal em RNs pré-termos submetidos à ventilação não invasiva com uso de prongas nasais.

\section{MÉTODO}

Foi utilizado um recurso da prática baseada em evidências, a revisão integrativa de literatura, a qual possibilita a construção de conhecimento em enfermagem, produzindo um saber fundamentado em evidências científicas aplicáveis à realização de uma prática clínica de enfermagem de qualidade ${ }^{(11-12)}$. Desta forma buscou-se responder ao seguinte questionamento: quais os cuidados de enfermagem para prevenção de lesão de septo nasal em RNs pré-termo em uso de pronga nasal?

Para a identificação e seleção das publicações foram utilizadas as bases de dados eletrônicas: Literatura Latino-Americana e do Caribe em Ciências da Saúde (LILACS); Base de Dados de Enfermagem (BDENF) e na biblioteca Scientific Eletronic Library on Line (SciELO); consultadas no site da biblioteca virtual em saúde (BVS); Cumulative Index to nursing and Allied health literature (CINAHAL) e US National Library of Medicine (PUBMED).

Foram utilizados os descritores em português catalogados nos Descritores em Ciências da Saúde (DeCS): Pressão positiva contínua em vias aéreas, Prematuro, Enfermagem, Cuidados de enfermagem, Ferimentos e lesões. $E$ em inglês os descritores catalogados no Medical Subject Headings (Mesh): Nasal continuous positive airway pressure, Premature, Neonate, Nasal septum perforation, Noninvasive ventilator, Newborns e Nursing care. Além desses foi utilizado o descritor não controlado Nasal necrosis. Para o cruzamento dos descritores foi empregado o operador booleano AND (Quadro 1). Nesse contexto, a busca em bases de dados procurou ser ampla e diversificada envolvendo as bases eletrônicas, busca manual em periódicos, as referências dos estudos selecionados, incluindo contatos com autores e material não publicado(12).

Como critérios de inclusão foram considerados artigos, dissertações e teses publicadas no período de 2000 a 2013 nas línguas portuguesa, inglesa e espanhola, que apresentassem cuidados de enfermagem na prevenção de lesão de septo nasal em neonatos em uso de ventilação não-invasiva com pronga nasal, disponibilizados on-line ou a partir de contato com o autor. Na busca dos artigos, 26 encontravam-se disponibilizados on line e apenas um foi conseguido por meio de contato com o autor. Foram excluídos os estudos referentes a revisões integrativas e sistemáticas, resumos de congressos, anais, editoriais e notas prévias. 
Quadro 1: Estratégias de busca utilizadas na base de dados BVS e PUBMED.

\begin{tabular}{|c|c|c|c|c|}
\hline Estratégia & $\begin{array}{l}\text { Base de } \\
\text { dados }\end{array}$ & $\begin{array}{l}\text { Cruzamento dos descritores } \\
\text { (descritores controlados) }\end{array}$ & $\begin{array}{l}\text { Cruzamento dos } \\
\text { descritores } \\
\text { (descritores não } \\
\text { controlados) }\end{array}$ & $\begin{array}{l}\text { Número de } \\
\text { estudos } \\
\text { encontrados }\end{array}$ \\
\hline $\mathrm{E} 1$ & SciELO & $\begin{array}{c}\text { Pressão positiva contínua em vias } \\
\text { aéreas AND Prematuro AND } \\
\text { Enfermagem }\end{array}$ & 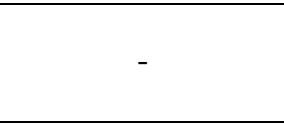 & 3 \\
\hline \multirow{3}{*}{ E2 } & SciELO & \multirow{3}{*}{$\begin{array}{c}\text { Pressão positiva contínua em vias } \\
\text { aéreas AND Prematuro AND Cuidados } \\
\text { de enfermagem AND Ferimentos e } \\
\text { lesões }\end{array}$} & \multirow{3}{*}{-} & 0 \\
\hline & LILACS & & & 9 \\
\hline & BDENF & & & 1 \\
\hline E3 & PUBMED & $\begin{array}{l}\text { Nasal continuous positive airway } \\
\text { pressure AND Premature }\end{array}$ & Nasal necrosis & 4 \\
\hline E4 & PUBMED & $\begin{array}{l}\text { Nasal continuous positive airway } \\
\text { pressure AND Neonate }\end{array}$ & Nasal necrosis & 7 \\
\hline E5 & PUBMED & $\begin{array}{c}\text { Nasal continuous positive airway } \\
\text { pressure AND Premature }\end{array}$ & Nasal necrosis & 1 \\
\hline E6 & PUBMED & $\begin{array}{c}\text { Nasal continuous positive airway } \\
\text { pressure AND Nasal septum } \\
\text { perforation AND Neonate }\end{array}$ & - & 0 \\
\hline E7 & PUBMED & Noninvasive ventilator AND Newborns & - & 2 \\
\hline E8 & CINAHAL & $\begin{array}{c}\text { Nasal continuous positive airway } \\
\text { pressure AND Nasal septum } \\
\text { perforation AND Neonate AND } \\
\text { Nursing care }\end{array}$ & - & 0 \\
\hline & & $\begin{array}{c}\text { TOTAL } \\
\end{array}$ & & 27 \\
\hline
\end{tabular}

Na seleção dos estudos foi realizada inicialmente a leitura dos títulos e resumos dos artigos, teses e dissertações, excluindo-se aqueles que não contemplaram os critérios de inclusão. Em seguida foi realizada a leitura na íntegra das publicações selecionadas.

$\mathrm{Na}$ busca dos artigos, teses e dissertações foram identificadas inicialmente 12 artigos na Biblioteca Virtual de Saúde (BVS) e 15 nos US National Library of Medicine (PUBMED), totalizando 27 artigos. Após leitura dos títulos e resumos foram selecionados 14 artigos. Destes, oito artigos foram da Literatura Latino-Americana e do Caribe em Ciências da Saúde (LILACS); um da BDENF, três da US National Library of Medicine (PUBMED) e dois estudos foram oriundos da biblioteca Scientific Eletronic Library on Line (SciELO). Na base de dados CINAHAL nenhum estudo foi encontrado. Os 13 estudos excluídos inicialmente, não respondiam a pergunta condutora e após leitura dos artigos na íntegra, dois não detalhavam o método utilizado no estudo, outro a população estudada não era de RN conforme critério de inclusão e dois eram estudos de revisão.

A descrição do processo de seleção das publicações encontra-se esquematizada na Figura 1.

A coleta de dados e avaliação do rigor metodológico dos estudos baseou-se em instrumento validado por Ursi ${ }^{(13)}$, intitulado Instrumento para coleta de dados, o qual contempla a identificação do estudo, suas características metodológicas, a avaliação do rigor metodológico, das intervenções realizadas e dos resultados obtidos. Somam-se a estes itens a identificação de limitações ou vieses de pesquisa.

Para avaliação do nível de evidência dos estudos foi utilizada uma hierarquia de evidência, segundo delineamento do estudo(14): nível 1 , refere-se as evidências resultantes da meta-análise de múltiplos estudos clínicos controlados e randomizados; nível 2, resulta de evidências de estudos individuais com delineamento experimental; nível 3, evidências de estudos quase-experimentais; nível 4, refere-se evidências de estudos descritivos, não-experimentais ou com abordagem qualitativa; nível 5 , provenientes de relatos de casos ou de experiência e o nível 6, baseadas em opiniões de especialistas. 
Bases de dados: LILACS; BDENF; PUBMED e na biblioteca SciELO.

Limites:

Estudos relacionados aos aspectos da assistência de enfermagem para a prevenção de lesão de septo nasal em neonatos em uso de prongas nasais

Disponibilização de texto on line ou a partir do autor

Publicações nos anos de 2000 a 2013, nos idiomas português, inglês e espanhol.

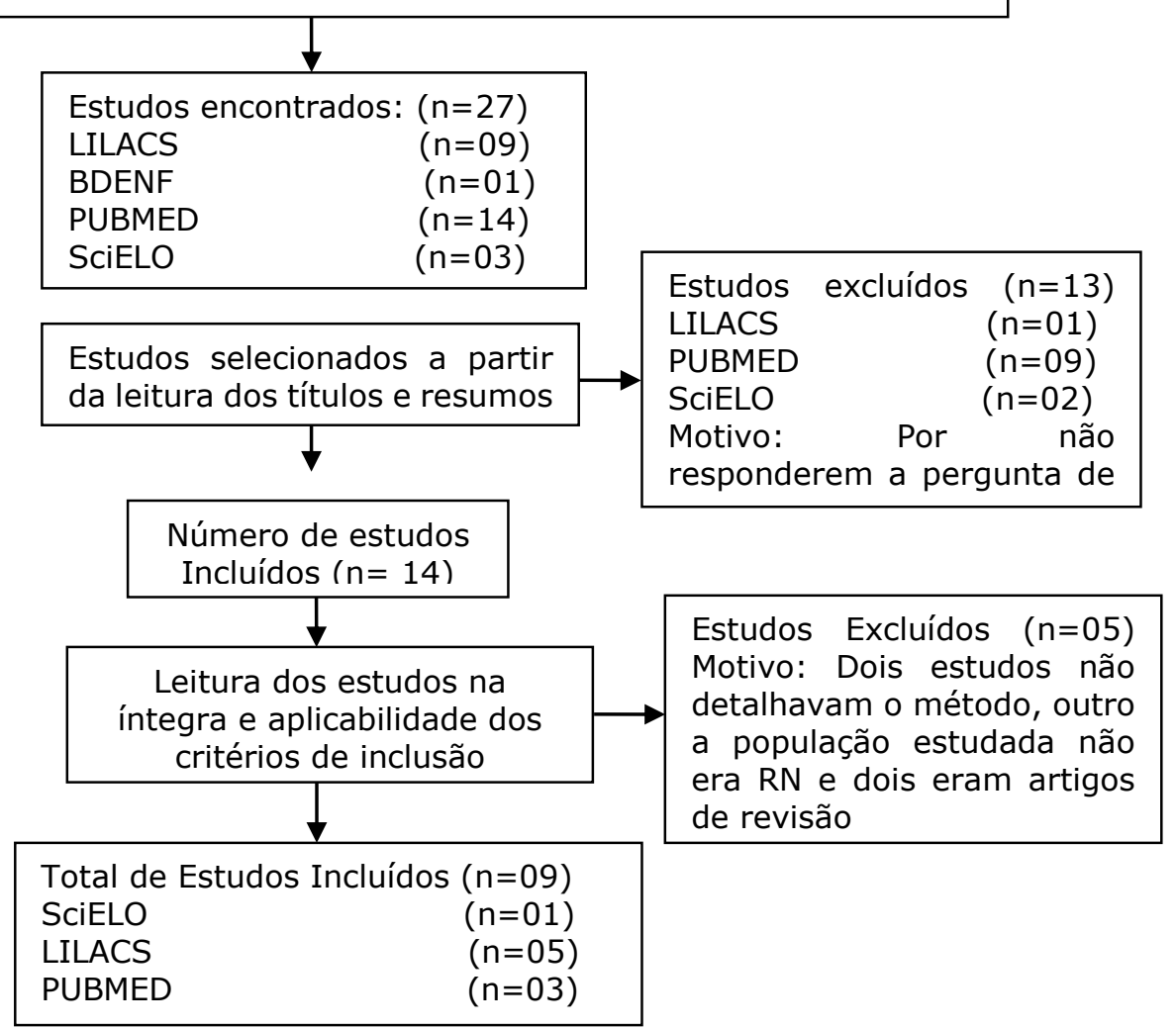

Figura 1: Fluxograma de seleção dos estudos.

\section{RESULTADOS}

Concernente aos tipos de estudo, foram evidenciados dois ensaios clínicos randomizados, dois estudos de coorte longitudinais, quatro estudos descritivos transversais e um estudo prospectivo série de casos, totalizando nove estudos. Com relação aos níveis de evidência encontrados, dois artigos se encontravam no nível de evidência 2 e sete artigos foram classificados no nível de evidência 4.

A partir da leitura dos estudos incluídos foram identificados os cuidados de enfermagem relacionados à prevenção de lesão de septo nasal por pronga. Esses resultados foram agrupados em dois subgrupos representados pelos cuidados relacionados à qualidade do material e aos cuidados prestados aos neonatos na instalação e manutenção da terapêutica. Tais aspectos foram igualmente evidenciados por estudiosos nos últimos dez anos( ${ }^{(7,15-16)}$.

Ressalta-se que dos nove estudos selecionados apenas um destacou limitação do estudo, relativo ao pequeno poder amostral. Outro aspecto se deve às características dos tipos de estudo, quatro artigos são provenientes de estudos transversais e uma série de casos que não permitem traçar relações de causa e efeito. A relação dos artigos de acordo com o autor, ano de publicação, país de origem, tipo de estudo, população, amostra, nível de evidência e resultados encontram-se no Quadro 2. 
Quadro 2: Artigos de acordo com autor, ano de publicação, país de origem, tipo de estudo, população, amostra, nível de evidência e resultados.

\begin{tabular}{|c|c|c|c|c|c|}
\hline \multirow[b]{2}{*}{$\begin{array}{c}\text { Autor, ano de } \\
\text { publicação e país }\end{array}$} & \multirow[b]{2}{*}{$\begin{array}{c}\text { Tipo de estudo; População e } \\
\text { variáveis estudadas }\end{array}$} & \multirow[b]{2}{*}{$\begin{array}{c}\text { Procedimento para coleta de } \\
\text { dados }\end{array}$} & \multirow[b]{2}{*}{$\begin{array}{l}\text { Nível de } \\
\text { evidência }\end{array}$} & \multicolumn{2}{|c|}{ RESULTADOS } \\
\hline & & & & $\begin{array}{l}\text { Cuidados de Enfermagem } \\
\text { relacionados ao material }\end{array}$ & $\begin{array}{l}\text { Cuidados de Enfermagem } \\
\text { relacionados ao neonato }\end{array}$ \\
\hline $\begin{array}{l}\text { Yong SC, Chen SJ, } \\
\text { Boo NY(8). 2005, USA }\end{array}$ & $\begin{array}{l}\text { Tipo: estudo randomizado controlado; } \\
\text { População: RNS de muito baixo peso } \\
\text { que receberam CPAP nasal com } \\
\text { máscara nasal }(\mathrm{n}=41 \text { ) e com pronga } \\
(\mathrm{n}=48) \text { no período de Julho de } 2001 \text { a } \\
\text { Dezembro de 2003; } \\
\text { Variáveis: Peso; IG; Pronga nasal; } \\
\text { máscara nasal; trauma nasal }\end{array}$ & $\begin{array}{l}\text { Acompanhamento diário dos RNS } \\
\text { randomizados para o uso de pronga } \\
\text { nasal e máscara; } \\
\text { Utilização de Instrumento de } \\
\text { avaliação clínica diária do septo } \\
\text { nasal dos Rns com pronga e máscara } \\
\text { nasal. }\end{array}$ & 2 & $\begin{array}{l}\text { Pronga nasal de tamanho } \\
\text { adequado para o peso do } \\
\qquad \text { RN; } \\
\text { Qualidade da pronga nasal } \\
\text { em material de silicone, } \\
\text { macio e com formato } \\
\text { anatômico. }\end{array}$ & $\begin{array}{c}\text { Proteção do septo nasal com placa } \\
\text { de hidrocolóide antes da instalação } \\
\text { da pronga; } \\
\text { Fixação e estabilização das } \\
\text { tubulações, sem tração; } \\
\text { Inspeção do septo nasal a cada } 3 \\
\text { horas. }\end{array}$ \\
\hline $\begin{array}{c}\text { Gunlemez A, Isken T, } \\
\text { Gocalp AS, Turker G, } \\
\text { Ansay EA }{ }^{(21)} \cdot 2009, \\
\text { USA }\end{array}$ & $\begin{array}{l}\text { Tipo de estudo: estudo prospectivo } \\
\text { controlado; } \\
\text { População: recém-nascidos pré-termo } \\
\text { em uso de CPAP. Grupo1 sem uso de } \\
\text { gel silicone ( } n=87 \text { ) e Grupo } 2 \text { com uso } \\
\text { do gel silicone ( } n=92) \text {. } \\
\text { Variáveis: Gel silicone; lesão nasal; } \\
\text { graus de lesões. }\end{array}$ & $\begin{array}{l}\text { Monitorização diária da área nasal de } \\
\text { neonatos em uso de CPAP nasal com } \\
\text { e sem proteção com gel silicone } \\
\text { Utilização de instrumento para o } \\
\text { registro do Tempo; início e duração } \\
\text { no uso do CPAP nasal. }\end{array}$ & 2 & - & $\begin{array}{l}\text { Proteção nasal com gel silicone } \\
\text { para a redução da incidência e a } \\
\text { severidade da lesão nasal; } \\
\text { Monitorização dos locais de pressão } \\
\text { da pronga nasal a cada } 3 \text { horas. }\end{array}$ \\
\hline $\begin{array}{l}\text { Ota NT, Davidson J, } \\
\text { Guinsburg R(19). } \\
\text { 2013, SP, Brasil }\end{array}$ & $\begin{array}{l}\text { Tipo: Estudo prospectivo, série de } \\
\text { casos. } \\
\text { População: Rns com IG }<37 \text { semanas e } \\
\text { peso }<1.500 . \\
\text { Variáveis: Pronga nasal;Grupo com } \\
\text { lesão;Grupo sem lesão; IG; peso; } \\
\text { sexo; Tempo de permanência e } \\
\text { indicação. }\end{array}$ & $\begin{array}{l}\text { Procedimento para coleta de dados: } \\
\text { Avaliação dos Rns admitidos na } \\
\text { terapêutica até o } 3^{\circ} \text { dia de uso nos } \\
\text { três turnos. Analisados aspectos } \\
\text { clínicos; característica e aplicação da } \\
\text { pronga nasal. }\end{array}$ & 4 & $\begin{array}{l}\text { Qualidade da pronga nasal } \\
\text { em material de silicone com } \\
\text { formato anatômico. }\end{array}$ & $\begin{array}{l}\text { Monitorização a do posicionamento } \\
\text { da pronga e do Rn a cada } 3 \text { horas } \\
\text { evitando trações. } \\
\text { Necessidade de adequação e } \\
\text { capacitação de pessoal relativo ao } \\
\text { uso do sistema. }\end{array}$ \\
\hline $\begin{array}{l}\text { Rego MAC, Martinez } \\
\text { FR(1). 2000, Brasil }^{(1)}\end{array}$ & $\begin{array}{l}\text { Tipo de estudo: prospectivo } \\
\text { longitudinal Grupos: recém-nascidos } \\
\text { com peso inferior a } 2.450 \mathrm{Kg} ; \mathrm{n}=96 \\
\text { Variáveis: Peso; idade gestacional; } \\
\text { CPAP nasal e complicações do CPAP. }\end{array}$ & $\begin{array}{l}\text { Utilização de Instrumento de } \\
\text { avaliação clínica diária; Boletim de } \\
\text { Apgar; Boletim de Silverman e } \\
\text { Anderson. }\end{array}$ & 4 & $\begin{array}{l}\text { Pronga nasal de tamanho } \\
\text { adequado para o peso do } \\
\qquad \mathrm{RN} ; \\
\text { Pronga nasal em material } \\
\text { de silicone, macio. }\end{array}$ & $\begin{array}{c}\text { Proteção do septo nasal com placa } \\
\text { de hidrocolóide antes da instalação } \\
\text { da pronga; } \\
\text { Fixação e estabilização das } \\
\text { tubulações, sem tração; } \\
\text { Umidificação e aquecimento do } \\
\text { sistema. }\end{array}$ \\
\hline
\end{tabular}




\begin{tabular}{|c|c|c|c|c|c|}
\hline \multirow[b]{2}{*}{$\begin{array}{c}\text { Autor, ano de } \\
\text { publicação e país }\end{array}$} & \multirow[b]{2}{*}{$\begin{array}{c}\text { Tipo de estudo; População e } \\
\text { variáveis estudadas }\end{array}$} & \multirow[b]{2}{*}{$\begin{array}{l}\text { Procedimento para coleta de } \\
\text { dados }\end{array}$} & \multirow[b]{2}{*}{$\begin{array}{l}\text { Nível de } \\
\text { evidência }\end{array}$} & \multicolumn{2}{|c|}{ RESULTADOS } \\
\hline & & & & $\begin{array}{l}\text { Cuidados de Enfermagem } \\
\text { relacionados ao material }\end{array}$ & $\begin{array}{l}\text { Cuidados de Enfermagem } \\
\text { relacionados ao neonato }\end{array}$ \\
\hline $\begin{array}{l}\text { Fischer C, Bertelle V, } \\
\text { Hohlfeld J, Forcada G } \\
\text { M, Stadelmann D C, } \\
\text { Tolsa JF( }{ }^{(9)} .2010 \text {, USA }\end{array}$ & $\begin{array}{l}\text { Tipo de estudo: estudo prospectivo; } \\
\text { População: recém-nascidos pré-termo } \\
\text { em uso de CPAP admitidos no período } \\
\text { de Janeiro de } 2002 \text { a Dezembro de } \\
2007 ; \\
\text { Variáveis: CPAP nasal; trauma nasal; } \\
\text { estágios da lesão nasal; IG; peso e } \\
\text { indicações. }\end{array}$ & $\begin{array}{l}\text { Acompanhamento diário da área } \\
\text { nasal de neonatos em uso de CPAP } \\
\text { nasal e avaliação do trauma nasal } \\
\text { Utilização de instrumento para o } \\
\text { registro de dados de identificação } \\
\text { demográficos e clínicos do RN. }\end{array}$ & 4 & - & $\begin{array}{c}\text { Monitorização dos locais de pressão } \\
\text { da pronga nasal a cada } 3 \text { horas; } \\
\text { Evitar pressão e friç̧ão do } \\
\text { dispositivo contra a narina do } \\
\text { neonato; uso de proteção com } \\
\text { placa de hidrocolóide no septo } \\
\text { nasal. }\end{array}$ \\
\hline $\begin{array}{l}\text { Antunes JCP } \\
\text { Nascimento MAL, } \\
\text { Gonas AVO, Araújo } \\
\text { MC, Cristoffol MM(15). } \\
\text { 2010, Brasil }\end{array}$ & $\begin{array}{l}\text { Tipo de estudo: estudo descritivo } \\
\text { observacional. } \\
\text { População: recém-nascido em uso de } \\
\text { CPAP nasal } \\
\text { Variáveis: CPAP nasal; efeitos } \\
\text { adversos do uso do CPAP nasal; } \\
\text { práticas de enfermagem para } \\
\text { prevenção das lesões nasais. }\end{array}$ & $\begin{array}{l}\text { Observação participante } \\
\text { Utilizado diário de campo para o } \\
\text { registro das observações realizadas. }\end{array}$ & 4 & $\begin{array}{c}\text { Qualidade da pronga nasal } \\
\text { em material de silicone, } \\
\text { macio e com formato } \\
\text { anatômico } \\
\text { Tamanho da pronga } \\
\text { adequada para o peso do } \\
\text { RN. }\end{array}$ & $\begin{array}{l}\text { Instalação do sistema de acordo } \\
\text { com protocolo; } \\
\text { Fixação e estabilização das } \\
\text { tubulações, sem tração; Proteção } \\
\text { do septo nasal com placa de } \\
\text { hidrocolóide antes da instalação da } \\
\text { pronga; Posicionamento da pronga } \\
\text { respeitando o eixo central do RN; } \\
\text { Manter o RN em posição } \\
\text { confortável no leito. }\end{array}$ \\
\hline $\begin{array}{c}\text { Silva DM, } \\
\text { Chaves EMC, Farias } \\
\text { LM, Lelis ALPA }^{(18)} \text {. } \\
\text { 2010, Brasil }\end{array}$ & $\begin{array}{l}\text { Tipo: estudo descritivo exploratório } \\
\text { População: Profissionais de } \\
\text { enfermagem } \mathrm{N}=30 \\
\text { Variáveis: Idade; sexo,tempo de } \\
\text { experiência; complicações referidas; } \\
\text { Intervenções informadas. }\end{array}$ & $\begin{array}{l}\text { Entrevistas com profissionais } \\
\text { utilizando questionário com questões } \\
\text { estruturadas e semi-estruturadas. }\end{array}$ & 4 & - & $\begin{array}{c}\text { Fixação e estabilização das } \\
\text { tubulações, sem tração; } \\
\text { Posicionamento do recém-nascido } \\
\text { com rolinhos de apoio; Realização } \\
\text { de massagem nasal de } 3 / 3 \text { hs }\end{array}$ \\
\hline $\begin{array}{l}\text { Medeiros SK, } \\
\text { Carvalho WB, Soriano } \\
\text { CF(24). 2012, RJ Brasil }^{(20)}\end{array}$ & $\begin{array}{l}\text { Tipo: estudo observacional seccional } \\
\text { do tipo inquérito; População: Todas as } \\
\text { instituições brasileiras de assistência } \\
\text { neonatal cadastrado no CNES. }\end{array}$ & $\begin{array}{l}\text { Levantamento utilizando-se } \\
\text { questionário relativo ao uso da } \\
\text { Ventilação com pressão positiva } \\
\text { intermitente nasal, enviadas aos } \\
\text { responsáveis pelos serviços. }\end{array}$ & 4 & - & $\begin{array}{l}\text { Utilização de proteção nasal com } \\
\text { placa de hidrocolóide para } \\
\text { prevenção da lesão de septo nasal. }\end{array}$ \\
\hline
\end{tabular}




\begin{tabular}{|c|c|c|c|c|c|}
\hline \multirow{2}{*}{$\begin{array}{c}\text { Autor, ano de } \\
\text { publicação e país }\end{array}$} & \multirow[b]{2}{*}{$\begin{array}{c}\text { Tipo de estudo; População e } \\
\text { variáveis estudadas }\end{array}$} & \multirow[b]{2}{*}{$\begin{array}{l}\text { Procedimento para coleta de } \\
\text { dados }\end{array}$} & \multirow[b]{2}{*}{$\begin{array}{l}\text { Nível de } \\
\text { evidência }\end{array}$} & \multicolumn{2}{|c|}{ RESULTADOS } \\
\hline & & & & $\begin{array}{l}\text { Cuidados de Enfermagem } \\
\text { relacionados ao material }\end{array}$ & $\begin{array}{l}\text { Cuidados de Enfermagem } \\
\text { relacionados ao neonato }\end{array}$ \\
\hline $\begin{array}{c}\text { Nascimento } \mathrm{R}, \\
\text { Ferreira AC, Coutinho } \\
\text { AC, Veríssimo, } \mathrm{RS}^{(16)} \text {. } \\
\text { 2009, Brasil }\end{array}$ & $\begin{array}{l}\mathrm{N}=147 \text { Neonatos pré-termos e termos } \\
\text { em VNI, Estudo descritivo Transversal. }\end{array}$ & $\begin{array}{c}\text { Coleta em protuários e utilizado } \\
\text { instrumento com questões } \\
\text { estruturadas. }\end{array}$ & 4 & $\begin{array}{l}\text { Pronga nasal de tamanho } \\
\text { adequado para o peso do } \\
\qquad \text { RN; } \\
\text { Qualidade da pronga nasal } \\
\text { em material de silicone, } \\
\text { macio e com formato } \\
\text { anatômico. }\end{array}$ & $\begin{array}{l}\text { Proteção do septo nasal com placa } \\
\text { de hidrocolóide antes da instalação } \\
\text { da pronga; } \\
\text { Fixação e estabilização das } \\
\text { tubulações, sem tração; } \\
\text { Inspeção do septo nasal a cada } 3 \\
\text { horas; Evitar reutilização do } \\
\text { dispositivo; } \\
\text { Empenho da equipe na utilização } \\
\text { do sistema; Necessidade de } \\
\text { capacitação de pessoal no uso da } \\
\text { terapêutica. }\end{array}$ \\
\hline
\end{tabular}




\section{DISCUSSÃO}

A análise dos estudos selecionados evidenciou que os cuidados de enfermagem direcionados à prevenção de lesão de septo nasal pelo uso de prongas são pautados na qualidade do material e na aplicação do método.

Nesse sentido, com relação aos aspectos relacionados diretamente ao dispositivo, as prongas nasais de pequeno calibre quando instaladas no neonato tornam-se móveis no interior da narina e podem necessitar de maior pressão, para evitar escape de ar e gerar a pressão

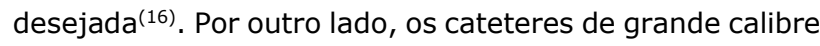
podem comprimir a narina. Nessas situações poderão ocorrer lesão da mucosa nasal e columela(16-17). A qualidade da pronga nasal, constituída de material siliconizado, com consistência macia foi descrita em cinco estudos $^{(1,8,15-16,18-19)}$; o tamanho da pronga adequada de acordo com o peso do RN, conforme escala indicada pelo fabricante, foi apontado em quatro dos nove estudos selecionados, como fator de proteção para o desenvolvimento de lesão de septo nasal(1,8,15-16,18).

$\mathrm{Na}$ realidade de muitas unidades de terapia neonatais brasileiras, o reaproveitamento de materiais é uma prática constante. Assim, existem evidências de que a pronga nasal reutilizável constitui fator de risco para o aparecimento de lesões nasais, baseado no fato de que o processo de desinfecção e esterilização aos quais tais dispositivos são expostos poderá estar desgastando o material, tornando-os menos flexíveis ${ }^{(16)}$. Desta forma, é recomendável que o dispositivo seja de uso único e descartável, aspecto evidenciado em dois estudos ${ }^{(1,16)}$.

Nos cuidados diretos ao neonato tem sido destacada a necessidade de inspeção da integridade da pele do recém-nascido pré-termo, especificamente da columela e septo nasal(8-9,16,20-21). Tal inspeção deverá ser feita a cada turno de trabalho, possibilitando o diagnóstico precoce de uma possível lesão. É destacada a importância da avaliação periódica da pele do recém-nascido, diagnosticando comprometimento cutâneo como risco de ruptura da pele entre outros, viabilizando a adoção de medidas profiláticas e fornecendo subsídios para a sistematização da assistência de enfermagem ${ }^{(15,21-22)}$.

A necessidade de proteção de septo nasal foi recomendada na maioria dos artigos analisados, ${ }^{(1,8-9,15-}$ 16,19-21). Sendo utilizada de preferência a proteção com hidrocolóide em placa, para evitar o atrito da pele com o dispositivo, contra a columela e o septo nasal(22). Paralelamente, conforme mostrado em estudo realizado, bons resultados foram encontrados com a utilização de proteção com gel silicone aplicado diretamente no septo nasal, o qual forma uma barreira protetora evitando o atrito entre o dispositivo e a columela nasal, constituindo alternativa, com comprovada eficácia para a redução de lesão nasal em pré-termos ${ }^{(21)}$.

Por ocasião da instalação do dispositivo, rotineiramente realizada pela equipe de enfermagem, cinco estudos ${ }^{(1,8,15-16,21)}$ recomendam que o procedimento seja executado procedendo o encaixe da pronga nasal com o duplo tubo curto voltado para a parte central da abertura nasal, sendo aplicado de maneira suave e evitando pressão demasiada.

As tubulações adaptadas a pronga, situadas horizontalmente, uma de cada lado da face e fixadas na touca, evitando repuxar a narina para cima ou para as laterais favorecem sua estabilização(7,23). Caso contrário, a tração e o peso exercidos pelas conexões sobre a pronga repercutirá em aparecimento de lesão nasal, sendo recomendada em cinco estudos a estabilização dessas tubulações ${ }^{(8-9,15-16,18)}$. A monitorização do posicionamento da pronga nasal a cada 3 ou 4 horas, foi ressaltada em três estudos e deverá constituir um procedimento rotineiro da equipe de enfermagem ${ }^{(9,15-16)}$, sendo o intervalo adaptado a rotina de cada serviço.

Da mesma forma, a umidificação das narinas e o aquecimento dos gases, constituem importante conduta, contribuindo para maior fluidez, facilitando remoção de secreções, cuidados recomendados em um estudo ${ }^{(1)}$. Tais procedimentos atuam como aspectos fundamentais para a prevenção de lesão de septo nasal no cuidar diário da enfermagem a essa clientela. É ressaltada ainda, uma maior cautela por ocasião das aspirações de vias aéreas superiores, tendo em vista a fragilidade nasal, sendo recomendada a utilização de sondas com calibres não superiores a $5 \mathrm{~mm}$, pelo risco de irritação e inflamação local ${ }^{(15,23)}$.

No que diz respeito à reatividade dos recém-nascidos pré-termo, mediante a diversidade de aparatos tecnológicos para sua monitorização, torna-se indispensável à adoção de um posicionamento adequado do neonato por meio de estratégias de conforto, em acordo com estudo recente referente ao protocolo para cuidados de enfermagem na prevenção de tais lesões ${ }^{(23)}$, como: uso de rolinhos, coxins e ninhos; isto manterá o neonato mais tranquilo evitando deslocamentos frequentes do dispositivo nasal, o que poderia desestabilizá-lo do ponto de vista respiratório e levar a perda da integridade da epiderme nasal devido ao atrito gerado $^{(15,18)}$.

É fato que pela fragilidade nasal do neonato prétermo, a pressão exercida pela pronga leva a isquemia local e favorece o aparecimento de necrose ${ }^{(7-8)}$. Por isso, 
soma-se aos cuidados de enfermagem proceder movimentos circulares no sítio de contato com o dispositivo em intervalos de três em três horas ${ }^{(9,15,18)}$. Em dois estudos foi reforçada a possibilidade de descanso do neonato do uso do método, por pelo menos 6 horas, de acordo com as condições clínicas do mesmo ${ }^{(8,22)}$. Para isso é recomendada à utilização de máscara nasal, sendo alternada por um período de 72 horas com a pronga nasal. Aspecto ressaltado em estudo envolvendo recém-nascidos de muito baixo peso, com complicações pelo uso de pressão positiva em vias aéreas(7).

Em se tratando da alta vulnerabilidade do recémnascido pré-termo, em adquirir infecções ligadas à assistência à saúde, e sendo a pele uma importante porta de entrada para agentes patogênicos, reforça-se a importância da lavagem das mãos antes e após todo e qualquer procedimento, aspecto evidenciado por um estudo $^{(15)}$.

\section{CONCLUSÃO}

Este estudo evidenciou que a prevenção de lesões nasais em recém-nascidos pré-termos em uso de prongas

\section{REFERÊNCIAS}

1. Rego MAC, Martinez FE. Repercussões clínicas e laboratoriais do CPAP nasal em recém-nascidos pré-termo. J. pediatr. $2000 ; 76(5): 339-48$.

2. Rocha $E$, Carneiro EM. Benefícios e complicações da ventilação mecânica não-invasiva na exacerbação aguda da doença obstrutiva crônica. Rev Bras Ter Intensiva. 2008; 20(2):184-9. 3. Millet V, Lacroze V, Bartoli JM. Unal D. Necrose de la columelle nasale secundaire à la pression positive continue (PCC) nasale. Arch Pediatr. 1997;4:485-88.

4. Pillow J J. Which Continuous Positive Airway Pressure System is best for Preterm infant with Respiratory Distress Sindrome? Clin Perinatol. 2012;39;483:96.

5. Buettiker V, Hug MI, Boenziger O, Meyer C, Frey B. Advanteges and disativanges of different nasal CPAP systems in newborns. Neonat Paediatr Intensive Care 2004;30:926-30.

6. MS (Ministério da Saúde). Atenção à saúde do recém-nascido: guia para profissionais da saúde. Brasília-DF; 2011. v. 3. 7. Robertson NJ, McCarthy LS, Hamilton PA, Moss ALH. Nasal deformities resulting from flow driver continuous positive airway pressure. Arch Dis Child. 1996 Aug;75:209-288.

8. Yong SC, Chen SJ, Boo NY. Incidence of nasal trauma associated with nasal prong versus nasal mask during continuous positive airway pressure treatment in very low birth weight infants: a randomized control study. Arch Dis Child Fetal Neonatal 2005;90:480-483.

9. Fischer C, Bertelle V, Holfeld J, Forcada-Guex M, StadelmannDiaw C, Tolsa JF. Nasal trauma due to continuous positive airway pressure in neonates. Arch Dis Child Fetal Neonatal. 2010 Nov;95(6):447-51.

10. Adriano LSM, Freire ILS, Pinto JTM. Cuidados intensivos com a pele do recém-nascido pré-termo. Rev. Eletr. Enf. [internet]. 2009 [cited 2011 Jul 4];11(1):173-80. Available from: http://www.fen.ufg.br/revista/v11/n1/pdf/v11n1a22.pdf. 11. Whittemore $\mathrm{R}, \mathrm{Knalfl} \mathrm{K}$. The integrative review: update methodology. Journal of Advanced Nursy 2005;52(3):546-53. 12. Galvão CM, Savada NO, Trevisan NA. Revisão sistemática: recurso que proporciona a incorporação das evidências na prática depende da qualidade e adequação do material utilizado, técnica de instalação das prongas nasais e monitorização dos cuidados de Enfermagem.

A utilização de prongas nasais em material de silicone, flexível, em formato anatômico, com tamanho de acordo com o peso do RN; a proteção nasal com placa de hidrocolóide; fixação adequada das tubulações e monitorização dos locais de pressão da pronga no mínimo a cada três horas constituem cuidados para redução da incidência e gravidade das lesões nasais nos prematuros.

Destacam-se os cuidados dispensados ao neonato durante a terapêutica, onde a necessidade da avaliação contínua por parte da equipe de enfermagem em todas as etapas do processo se reveste de fundamental importância.

Desta forma, a relevância desse estudo para a prática da enfermagem está pautada na adoção de práticas que minimizem os efeitos do método de ventilação não invasiva sobre os neonatos pré-termo, decorrentes de mudanças de comportamento e do constante empenho da equipe, na escolha do material, na adequação da técnica e nos cuidados diretos ao neonato.

da enfermagem. Rev Latino-Am Enfermagem 2004;12(3):54956.

13. Ursi ES, Galvão CM. Prevenção de lesões de pele no perioperatório: revisão integrativa da literatura. Rev. Latino-am Enfermagem 2006;14(1):124-31.

14. Startler CB, Morsi D, Rucki S, Broughton S, Corrigan B, Fitzgerald J et al. Utilization-focused integrative reviews in a nursing service. Appl Nurs Res. 1998;11(4):195-206.

15. Antunes, JP. Tecnologia Secundária el Tratamiento del Recién nacido prematuro. Rev. Eletr Cuatrimestral de Enfermaria. 2010;20:4-5.

16. Nascimento RM, Ferreira AL, Coutinho AC, Veríssimo RC. Frequência de lesão nasal em neonatos por uso de pressão positiva contínua nas vias aéreas com prong. Rev. Latino-am Enfermagem 2009;17(4):489-94.

17. Robert MB. Nasal continuous positive airway pressure for the respiratory care of the newborn infant. Rev. Resp.Care 2009;54(9):1209-235.

18. Silva DM, Chaves EMC, Farias LM, Lelis ALPA. Uso de Pressão Positiva Contínua das vias aéreas em recém-nascidos: conhecimento da equipe de enfermagem. Rev. Rene 2010; 11(Especial): 195-203.

19. Ota N T, Davidson J, Guinsburg R. Lesão nasal precoce pelo uso da pronga nasal em recém-nascidos prematuros de muito baixo peso: estudo piloto. Rev. bras.ter.intensiva (São Paulo). 2013; 25(3):245-250. Disponível em : http://dx.doi.org/10.5935/0103-507X.20130042.

20. Gonzaga CS, Silva DCB, Alonso CFR, Oliveira A C, Torreão LA, Troster EJ. Ventilação não-invasiva em crianças com insuficiência respiratória aguda: Uma revisão sistemática da literatura. Einstein, São Paulo;2011;9(1):90-4.

21. Günlemez A, Isken T, Gökalp AS, Turker G, Arisoy EA. Efeitos gel silicone nas injúrias nasais associado com o CPAP nasal. Indian Pediatrics 2010;47(3):265-7.

22. Squires $A J$, Hyndman $M$. Prevention of nasal injuries secondary to NCPAP application in the ELBW infant. Neonatal Netw. $2009 ; 28(1): 13-27$. 
23. McCoskey L. Nursing Guidelines for prevention of nasal breakdown in neonates receiving nasal CPAP. Adv Neonatal Care 2008;8(2):116-24.

24. Medeiros SK, Carvalho WB, Soriano CF. Practices of use of nasal intermittent positive pressure ventilation (NIPPV) in neonatology in northeastern Brazil. J Pediatr (Rio J).

2012;88(1):48-53.

Artigo recebido em 30/11/2012.

Aprovado para publicação em 07/02/2014.

Artigo publicado em 30/06/2014. 